\title{
Hyponatremia, osmotic demyelination syndrome, and the importance of the patient's history
}

\author{
Dominique G. Gagnon MD, PhD
}

\begin{abstract}
Central pontine myelinolysis (CPM), first described in 1959, is a symmetrical non-inflammatory demyelinating disease with loss of oligodendrocytes that occurs most often following a rapid correction of severe hyponatremia (i.e., $<120 \mathrm{mmol} / \mathrm{L}$ ). It presents as a biphasic disease with initial seizure or encephalopathy, followed by clinical improvement and subsequent rapid deterioration with bulbar dysfunction, oculomotor dysfunction, various degree of paresis, and even locked in syndrome. Its occurrence is rare $\approx 0.6 \%$ of severe hyponatremia), it is diagnosed clinically and confirmed with brain imaging, ideally with magnetic resonance image, and it is reversible in approximately half the patients. Lesions are classically identified in the pons but extra pontine lesions (in basal ganglia, cerebellar white matter, thalamus, and hippocampus) have also been identified. The most commonly accepted molecular mechanism involves brain cell volume regulation with a rapid shift of osmole following brain edema which establishes during the chronic hyponatremic phase. For these reasons, osmotic demyelination syndrome (ODS) is a better term. The most identified risk factor is severe hyponatremia, but other electrolyte abnormalities can contribute, in particular, if the patient is an alcoholic or malnourished. This diagnosis should also be suspected in post-op patients with nausea and headache non-responsive to antiemetic and analgesic drugs. An essential step is an appropriate medical history, a list of medications, physical examination, and basic initial lab tests with the goal of identifying possible easily reversible causes of hyponatremia. Correction of severe hyponatremia with neurological symptoms should be done using rapid boluses of hypertonic saline solution in rapid succession with goals of increasing serum sodium by 5-6 mEq/L in the first two hours, which should be stopped if the level has risen by $10 \mathrm{mEq} / \mathrm{L}$ in the first five hours, and with the overall correction goal not to exceed $15-20 \mathrm{mEq} / \mathrm{L}$ in 48 hrs. This method has been shown safe in all hospital settings studied. Serial measurements of electrolyte levels and neurological examinations are recommended, as are correction of all electrolyte abnormalities, in particular magnesium and potassium. Thiamine should be given to all patients with chronic alcohol use who present with hyponatremia and encephalopathy.
\end{abstract}

Keywords: hyponatremia, central pontine myelinolysis, osmotic demyelination syndrome, alcohol use disorder, electrolytes abnormalities

\section{INTRODUCTION}

Hyponatremia is arguably the most common electrolyte disorder in hospitalized patients. ${ }^{1-5}$ In the

Corresponding author: Dominique Gagnon

Contact Information: Dominique.Gagnon@ttuhsc.edu DOI: 10.12746/swrccc.v9i37.797 general population the prevalence is estimated at $1-2 \%$; with women, the elderly, and those with comorbidities being more at risk. ${ }^{3}$ Hyponatremia has been associated with a wide variety of adverse outcomes: delirium, falls, prolonged hospitalizations, and death. A U-shape relationship was found between relative hazard ratio for all-cause mortality and serum sodium levels, and a quasi linear relationship between duration of hyponatremia in months and mortality. ${ }^{2,3}$ While 
the exact ranges of serum sodium levels can vary slightly, mild hyponatremia can be defined as serum sodium ranging from $130-135 \mathrm{mmol} / \mathrm{L}$ and is generally asymptomatic. Non-specific symptoms, such as fatigue, malaise, nausea, and unsteadiness, may start to be noticed in patients with serum sodium levels ranging from $120-130 \mathrm{mmol} / \mathrm{L} .{ }^{1}$ Severe hyponatremia, i.e., serum sodium level $<125 \mathrm{mmol} / \mathrm{L}$ (though a majority of the ODS cases reviewed had sodium levels $<120 \mathrm{mmol} / \mathrm{L}$ ), can be asymptomatic, provoke headaches, render patients lethargic or obtunded, and can lead to severe neurological symptoms, such as seizures, coma and respiratory arrest requiring immediate attention. ${ }^{2,3,5,6}$ Overly rapid correction of hyponatremia, i.e. a rate faster than $15-20 \mathrm{mmol} / \mathrm{L} /$ day, has been linked with development of central pontine myelinolysis (CPM), renamed osmotic demyelination syndrome (ODS), which can be fatal. To our knowledge and to this date, there is no risk calculator for ODS, but a calculator for prediction of overcorrection of severe hyponatremia has recently been proposed. ${ }^{7}$ This review considers and discusses historical cases, clinical studies and risk factors, the physiology and pathophysiology of ODS, and guidelines for correction of severe hyponatremia, and finally hyponatremia in the context of an alcohol use disorder due to the higher risk for ODS in this patient population.

\section{Central pontine myelinolysis HISTORICAL PERSPECTIVE}

The first known cases of a "new demyelinating disease" named central pontine myelinolysis (CMP) were described in 1959 by Adams and colleagues. ${ }^{8}$ The affected patients were alcoholic and/or malnourished. Half of them exhibited symptoms, such as quadriplegia and pseudobulbar palsy, and the others were relatively asymptomatic. Pathology reported "myelin sheaths of all the nerve fibers in the central part of the basis pontis had been destroyed in a single, large, symmetric focus." Similar cases are frequently encountered in the ICU setting. These cases illustrate many of the now better known and identified risks factors and clinical course of this disease, which was later renamed osmotic demyelination syndrome (ODS).
Case 1 was an alcoholic man hospitalized for delirium tremens. On admission, he was septic and hypotensive. He was found to have pneumonia (dullness to percussion, increased bronchial breath sounds with egophony, and fine inspiratory rales in his left lower lobe, WBC $11,000 / \mu \mathrm{L}$, and a CXR with left lower lobe consolidation); he had normal cranial nerve examination but his deep reflexes were absent below the knees. He was treated with penicillin, and he received $3 \mathrm{~L}$ of saline daily. On day 5 , he had a fever of $105^{\circ} \mathrm{F}$, became confused, and had worsening leukocytosis. He appeared to have difficulty swallowing which was attributed to his mental status. He then received tetracycline and improved, but his swallowing difficulties worsened. An ECG showed a QT interval of $350 \mathrm{~ms}$ on day 11 of hospitalization, and serum potassium was low at $3.1 \mathrm{mEq} / \mathrm{L}$, which was corrected with $30 \mathrm{meq} \mathrm{KCl}$. His quadriplegia and difficulty swallowing worsened, and he ended up with total paralysis and loss of all $\mathrm{CN}$ reflexes and respiratory drive prior to death from respiratory failure on day 22 .

Case 2 was an alcoholic and malnourished woman with altered mental status hospitalized for severe dehydration and anemia. Prior to hospitalization, she had been drinking heavily and eating poorly, had vomited extensively, and had intractable hiccups. On examination, she was uncooperative but had normal vital signs except for hypotension. Her skin was pale, her nail beds cyanotic, her tongue was dry and red, her lips dry and fissured, and she had hepatomegaly; her neurological examination was normal. A urine culture showed E. coli. She was treated with antibiotics and received dextrose and saline infusions. Her overall status improved until day 12 when she became confused, restless, and incontinent of urine and complained of calf and foot pain. On day 18, she was unable to speak, and her neurological examination was relevant for impaired swallowing, absent gag reflex, inability to protrude her tongue, neck muscle weakness, and severe flaccid weakness of all limb muscles. Her DTRs were absent, and the plantar responses were abnormal. Her sensory examination was intact. She continued to deteriorate, became completely quadriplegic on day 26 , and had paralysis of the intercostal muscles. Knee and ankle jerks were still absent, but she had a positive Babinski sign. She died on day 39 of hospitalization. 
Case 3 was a malnourished alcoholic man who drank 15-20 glasses of beer daily. He was admitted for difficulty walking and lower extremities weakness, in addition to a persistent, productive cough and intermittent fever. On admission, he was also septic likely due to pneumonia and had a dry and beefy-red tongue, absent reflexes below the knee, and a positive Babinski sign bilaterally. The details of his treatment were not stated in the report. It was concluded that he had a long-standing Wernicke's disease and tuberculosis concurrent with but not causing the illness. He died 11 days after admission.

In all three cases, post mortem pathology identified symmetrical lesions involving the base of pons and involving demyelination of all nerve tracts, which seemed to have originated from the center and spread radially. These lesions were remarkable for lack of oligodendrocytes. Nerve cells and the axis cylinder were preserved, and blood vessels were free of disease in all cases. Other demyelinating diseases, such as multiple sclerosis, were considered as possible causes but excluded based on one pathological feature, namely, inflammation was absent in the three cases.

These historic cases still to this date reflect the most common risk factors for CMP. While in none of these cases was the serum sodium level reported neither was its rate of correction, detailed neurological examinations were reported and are still relevant to this date. Since its original description in the late 1950s; knowledge of CMP has increased significantly as has our management of critically ill patients with sepsis and electrolyte abnormalities as discussed below. ${ }^{6,9,10}$

\section{Clinical studies}

Between 1964 and 1996, the diagnosis of CMP depended on clinical information and was essentially confirmed with necropsy/pathology. After its original description, the most common identified risk factor for development of CPM was hyponatremia, and CMP is essentially a disease of chronic hyponatremia. ${ }^{6,9,10}$ Rapid correction of acute hyponatremia (e.g., in marathon runners, ecstasy use) has virtually no risk for ODS. ${ }^{11}$ Patients presented with encephalopathy $(45 \%)$ followed equally frequently by seizures $(12.5 \%)$ and paresis $(12.5 \%),{ }^{6,9,10}$ The affected patients were more frequently alcoholic (54\%), cirrhotic, or status post liver transplantation (12-17\%), and to a lesser extent patients with renal failure, known neoplasm, or malnutrition. ${ }^{6,9,10}$ It was hypothesized that an osmole shift happening after a rapid correction of hyponatremia increased the risk of developing CPM, which was renamed ODS due to pathophysiology and to the fact that lesions can be present outside of the pons.

Since the mid-1980s, the standard approach for diagnosis of CPM uses radiological analysis, magnetic resonance imaging (MRI) being the most sensitive. Studies have confirmed that the most common risk factor for CPM is hyponatremia (66\%), and that the most common clinical presentation is encephalopathy $(37 \%)$ followed by seizures $(25 \%)$ and paresis $(25 \%)$ consistent with earlier reports. ${ }^{6,9,10}$ The patient population described included alcoholics (52\%), cirrhotic patients, patients with liver transplants (8-14\%), patients with renal failure, and malnourished patients who had an osmole shift (rapid correction of hyponatremia). ${ }^{6,9,10}$ It was estimated that about $52 \%$ recovered symptomatically, $25 \%$ died, and $23 \%$ had variable disability. ${ }^{6,9,10}$

Recently, 1,490 cases of severe hyponatremia $(<120 \mathrm{mEq} / \mathrm{L})$ were reviewed at the Geisinger Health System, PA, from 2001 to $2017 .{ }^{9}$ Osmotic demyelination syndrome diagnosed by brain MRI had a very low incidence $(0.6 \%$, i.e., 9 patients out of 1,490$)$, was nearly always associated with some episode of rapid correction of hyponatremia (7/9 cases), and was often also associated with other factors, such as alcoholism (6/9 cases), hypokalemia, and malnutrition. In that study, five patients had good neurological recoveries from the osmotic demyelination. Overall, occurrence of CPM was very rare, $0.6 \%$ with $50 \%$ recovery. This very low incidence rate has been confirmed by a Swedish nationwide study. ${ }^{12}$

In 1994, Lorh reported a case of ODS associated with lung cancer admitted with concurrent hypokalemia $(<2.8 \mathrm{mmol} / \mathrm{L})$ and hyponatremia $(<115 \mathrm{mmol} / \mathrm{L}) .^{13} \mathrm{He}$ reviewed 135 ODS cases diagnosed clinically or radiologically following a rapid correction of hyponatremia $(<126 \mathrm{mmol} / \mathrm{L}) ; 74$ had reported serum potassium and sodium levels at initial presentation, and about 
half had concurrent hyponatremia and hypokalemia $(<3.5 \mathrm{mmol} / \mathrm{L})$. Only 20 had serial measurements of serum sodium and potassium levels, and none had resolution of hypokalemia prior to the correction of hyponatremia. Of note, of the eight ODS patients with normal potassium levels, 4 had adrenal insufficiency, and 3 had renal failure. Consistent with other reports, a large fraction of the patients (40/74) survived, some with complete recovery and some with variable but persistent neurological deficits. The author suggested that hypokalemia concurrent with hyponatremia is more likely in volume depleted patients or in patients on diuretics, and that these patients are more likely to undergo a rapid correction of their sodium levels thus leading to ODS. This is due to reversible impaired water excretion, which has various identifiable and correctable causes, such as hypovolemia, thiazide use, drug-, stress-, or nausea-induced SIADH, hypoxia, and cortisol deficiency.

Other sporadic cases of ODS have been described and associated with other minor risk factors. Those include metabolic acidosis, hypophosphatemia, hypokalemia, diabetic ketoacidosis, renal failure/hemodialysis. ${ }^{13-18}$ The importance of these other risk factors is highlighted by some rarer cases of ODS occurring after an appropriate rate of serum sodium level correction. ${ }^{17,19}$ Unfortunately, these risk factors were not identified in a large cohort study. The low incidence of ODS over the years probably contributes to low quality supporting evidence for minor risks factors.

Osmotic demyelination syndrome is even less common in pediatric patients with only 106 cases reported in the literature between 1960 and $2018 .^{20}$ Those cases, similar to adults, were thought to occur after an overly rapid correction of hyponatremia ( $>12 \mathrm{mEq} / \mathrm{l}$ over $24 \mathrm{hrs}$ ). Clinical presentation is highly variable as for adults with some neurological symptoms (dysarthria, bulbar or ocular focal defects, paresis etc.). Of note, extrapontine lesions were also found in children and often presented with non-specific symptoms, such as altered mentation, seizure, deafness, altered gait, etc. Most cases occurred in children between age 1-5; up to $60 \%$ had either a complete or partial recovery, and $39 \%$ died. However, the number of children who passed from ODS after the year 2000 is astonishingly lower at $9 \%$.

\section{Presentation and diagnosis}

The classic clinical presentation is a male patient with liver cirrhosis, encephalopathy, and seizures found to have severe hyponatremia on initial lab tests. Symptoms can occur 1 to 14 days following the trigger, usually an osmole shift after a rapid correction of hyponatremia. ${ }^{10}$ The disease evolves typically in a biphasic manner: 1) seizure or encephalopathy, followed by 2) clinical improvement, then 3 ) rapid deterioration with bulbar dysfunctions, such as dysarthria, dysphagia, oculomotor dysfunction, various degrees of paresis, and even locked in syndrome, the most feared complication of rapid correction of hyponatremia. Fortunately, total or near total remission can occur in up to $50 \%$ of the cases. ${ }^{6}$ This is due to improvement in clinical diagnosis (e.g., improved laboratory studies which lead to identification of electrolytes abnormalities) and recognition of risk factors during recent decades. Liver transplant patients have lower chances of recovery from ODS, and this outcome is unlikely to change until the pathophysiology becomes better understood. ${ }^{6}$

A brain MRI is considered the gold standard for diagnosing CPM with pontine demyelination being the hallmark. Extrapontine lesions are seen in 13\% of cases; combined pontine and extra pontine lesions also occur in some cases, including in the basal ganglia (especially in the striatum, 34\%), cerebellar white matter $(33 \%$ to $55 \%)$, thalamus, or hippocampus. In those cases, osmotic demyelination syndrome might be a more appropriate denomination. 6,10,21 Unfortunately, and perhaps more troublesome, is that the absence of identified lesions on MRI does not exclude the diagnosis, as its radiologic evidence can be delayed by 1-2 weeks. ${ }^{6,10,21}$

\section{Physiology and pathophysiology}

The most serious complication of hyponatremia is cerebral edema (cell swelling) and a rapid osmole shift extracellularly following an overly rapid correction of chronic hyponatremia leading to hyponatremic encephalopathy and ODS.22 Diagnosing hyponatremic encephalopathy is difficult due to its vague and non-specific symptoms, such as headache, nausea, 
vomiting, which vary from patient to patient and might not always correlate with the severity of the hyponatremia. ${ }^{2,3}$ However, symptoms can rapidly progress to coma, seizure, and neurogenic pulmonary edema that can lead to respiratory arrest or trans-tentorial herniation. Post-operative patients, water intoxication, use of ecstasy, and thiazide diuretics ${ }^{22}$ are risk factors for the development of hyponatremic encephalopathy. Since post-operative nausea and vomiting are common, when these symptoms are associated with a headache and do not respond to antiemetics and analgesics, physicians should suspect hyponatremia and check serum sodium levels. These symptoms can be preludes of cerebral edema and herniation.

Hyponatremia is defined as serum sodium levels $<135 \mathrm{mmol} / \mathrm{L}$. It is due to a relative excess of body water compared with the total body stores of sodium and potassium. Urine production by the kidneys is essential to regulation of total body water content and affects, in return, serum level of sodium. The ability to produce concentrated urine and conserve water depends on hormonal control (by $\mathrm{ADH}$ ) of aquaporins at the luminal membrane in the collecting duct, ${ }^{23}$ a regulation pathway that depends on a CAMP and protein kinase $\mathrm{A}$.

Total osmolality and effective osmolality or tonicity have distinct effects on water balance and ion movement across biological membranes. Since only effective solutes create osmotic pressure gradients, only effective solutes lead to movement of water across cell membranes. Acute or chronic changes in osmotic pressure can lead to cell swelling and edema.

Many membrane proteins have been involved in cell volume regulation. The complete molecular mechanisms for cell volume regulation are not fully understood, even less for rapid changes, but a number of well-known membrane proteins are involved:24-29 the $\mathrm{Na}^{+} / \mathrm{K}^{+}$ATPase (also called the "pump"), the $\mathrm{Na}^{+} / \mathrm{H}^{+}$ exchanger, the $\mathrm{Na}^{+} / \mathrm{K}^{+} / 2 \mathrm{Cl}^{-}$and $\mathrm{K}^{+} / \mathrm{Cl}^{-}$cotransporters, several $\mathrm{K}^{+}, \mathrm{Cl}^{-}$channels, some of which respond to intracellular calcium or membrane tension, and the water channels aquaporins. The $\mathrm{Na}^{+} / \mathrm{K}^{+}$pump (which utilizes intracellular ATP to generate the $\mathrm{Na}^{+}$ transmembrane gradient) is undoubtedly important. Magnesium is essential for proper functioning of the
$\mathrm{Na}^{+} / \mathrm{K}^{+}$pump that generates the sodium gradient across cellular membrane. In addition, the lack of potassium or ATP may slow down the pump, which in turn will impair other sodium-dependent secondary transport of essential elements, such as inorganic and organic osmolytes (namely inorganic phosphate, glucose, etc.). Some of these channels and transporters are the targets of drugs commonly used. For example, antiarrhythmic drugs often target non-specifically various calcium, sodium and potassium channels, digoxin inhibits the $\mathrm{Na}^{+} / \mathrm{K}^{+}$pump, furosemide inhibits the $\mathrm{Na}^{+} / \mathrm{K}^{+} / 2 \mathrm{Cl}^{-}$channel in the thick ascending loop of Henle, etc. In addition, membrane protein expression, function, and regulation might be affected by acute and chronic changes of osmolytes in a cell-dependent manner, which explains why complete brain adaptation to hyponatremia takes $36-48$ hours.

\section{GUIDELINES FOR CORRECTION OF SEVERE HYPONATREMIA}

Due to many factors, including fear of ODS, hyponatremia is often mismanaged in the hospital. ${ }^{30,31}$ The first essential step is an appropriate history that includes medication, physical examination, and basic initial lab tests to lead to an accurate diagnosis that will often guide the proper course of treatment. Mild hyponatremia has been shown to respond well to fluid restriction, infusion of normal saline, or treating the underlying cause (whether it is due to a medication or another treatable medical condition, which invariably will not lead to a uniform algorithm) ${ }^{32}$ Thereafter, the patient's fluid status (euvolemic vs hypo- or hypervolemic) must be assessed as a criterion for the classification of hyponatremia. ${ }^{33,34}$ One should identify and treat any easily reversible cause of the hyponatremia, such as kidney failure, congestive heart failure, cirrhosis, nephrotic syndrome, adrenal and renal insufficiency, hypothyroidism, psychiatric disorder, or drug-induced hyponatremia. ${ }^{35}$ Numerous drugs are commonly associated with hyponatremia and include thiazide and loop diuretics, antipsychotic and antiepileptic medications, and calcium channel blockers, etc. $^{35}$ In some patient, ADH is constitutionally inappropriately secreted despite low serum sodium levels, leading to chronic hyponatremia. Vasopressin 
antagonists (so called "vaptans") have been used as a possible treatment of hyponatremia in the context of SIADH when water restriction has failed. ${ }^{36,37}$ Finally, identifying possible confounders, such as osmotically inactive solutes (glucose, alcohol), should be considered.

Complex algorithms have been proposed to manage severe hyponatremia and often lead to various approaches in different institutions. In the absence of a risk calculator for ODS supporting decision making, the proper correction method will remain variable. Recently, a risk factor calculator for overcorrection of severe hyponatremia has been proposed, and it includes factors, such as age, symptomatology, volume status, severity of sodium and potassium levels, urine osmolality, and presence or absence of a chest tumor. ${ }^{7}$ Most efforts have been mostly focused of preventing ODS rather than treatment, but the concept of "re-introduction of hyponatremia" when overcorrection has happened may prevent myelinolysis. ${ }^{38}$

An optimal treatment of severe hyponatremia, defined as a $\mathrm{Na}^{+}<120 \mathrm{mmol} / \mathrm{L}$ with neurological symptoms, was adopted by the International ExerciseAssociated Hyponatremia Consensus Development Congressand the European Clinical Practice Guidelines and several societies, including the European Society of Intensive Care Medicine (ESICM), the European Society of Endocrinology (ESE), and the European Renal Association - European Dialysis and Transplant Association (ERA-EDTA). ${ }^{39-41}$ Treatment consists of rapid boluses of $100-150 \mathrm{ml}$ of hypertonic $3 \%$ saline solution (osm $513 \mathrm{mEq} / \mathrm{L}$ ) in rapid succession with goals of increasing serum sodium by $5-6 \mathrm{mEq} / \mathrm{L}$ in the first 1-2 hrs. The hypertonic saline infusion should be stopped if the level has risen by $10 \mathrm{mEq} / \mathrm{L}$ in the first $5 \mathrm{hrs}$, and the overall correction should not exceed $15-20 \mathrm{mEq} / \mathrm{L}$ in $48 \mathrm{hrs}$. It is valid for use in all types of hyponatremia, i.e., if hyponatremia is moderate or severe, is acute or chronic, and is associated with moderate or severe hyponatremic encephalopathy. Vaptans have been considered by expert panel committees (European Society of Intensive Care Medicine, the European Society of Endocrinology, and the European Renal Association-European Dialysis and Transplant Association) not to be a valid treatment option for euvolemic hyponatremic patient with moderate to severe symptomatology. ${ }^{36}$

Despite some misconceptions, hypertonic saline can be administered by peripheral line and in a nonICU context. ${ }^{39}$ Indeed, it has been incorrectly stated that infusion of hypertonic saline is painful via peripheral line. Also, some institutions prefer the ICU setting for its infusion to have staff available for frequent assessment of neurological status. Finally, the use of hypertonic saline boluses does not pose higher risk than other methods (fluid restriction, normal saline).

Despite not being explicitly stated in the guidelines, serial measurement of serum electrolyte levels is critical to assess the rate of correction and reassess the proper course of action. Correction of all electrolyte abnormalities should be done as well; in particular, potassium, an effective osmole, should be corrected promptly as a deficit or excess can lead to cardiac arrhythmias.

\section{SPECIFIC POPULATION: alCOHOLIC PATIENTS}

Chronic alcohol consumption increases electrolyte abnormalities in several ways, from poor oral intake to renal and hepatic dysfunction. ${ }^{41}$ Chronic alcohol use can lead to liver cirrhosis, which adds a risk factor for CMP upon correction of hyponatremia.

In ER or ICU settings, commonly, alcoholic patients present with nausea, abdominal pain, and emesis and are found to have anion-gap metabolic acidosis on initial labs. In these cases, metabolic acidosis is specified as alcoholic ketoacidosis is a consequence of 1) diversion of the metabolism in favor of mobilization and delivery of long chain fatty acids to the liver, 2) decreased insulin/glucagon ratio, 3) increase in NADH/NAD ${ }^{+}$ratio, all of which favor the ketogenic state but also create an inhibitory effect on hepatic gluconeogenesis that can predispose patients to hypoglycemia, which can be life threatening.

Acute alcohol effects decrease ADH (vasopressin) levels, decreasing the amount of aquaporins channels at the luminal membrane in renal collecting duct, which increases clearance of free water. It thus increases urine volume production, decreases urine osmolality, and subsequently increases serum osmolality. This 
can cause hypernatremia and lower the intravascular volume. However, in chronic alcohol intake, there is an adaption to lower ADH levels, and hyponatremia is thought to be secondary to poor diet and oral electrolytes intake.

Other electrolyte abnormalities are also commonly associated with alcoholism, and those include hypophosphatemia. This is typically due to poor dietary intake of phosphate-rich food but can also be secondary to metabolic acidosis leading to an increase in renal excretion of phosphate. ${ }^{41}$ Phosphate is essential for the generation of ATP, the energy of the cells, and its depletion favors lactic acid production and ischemia. As mentioned above, alcohol-induced tubular dysfunction and alcohol-induced myopathy which promote increased phosphorus renal excretion and release from the muscle, respectively. ${ }^{41,42}$ Hypokalemia is multifactorial; it can be due to poor dietary intake, GI loss, impaired renal reabsorption and is worsened by acidosis and other electrolytes abnormalities, in particular hypomagnesemia. Indeed, increased potassium wasting by the ROMK (generating outward movement of potassium) channels occurs with reduced magnesium levels leading to decreased inhibition. ${ }^{43}$ Intracellular potassium is essential for muscular and neuronal activity; therefore, its repletion is crucial.

Divalent ions, such as calcium and magnesium, act to stabilize cellular membrane potential, inhibit inward rectifier potassium channels, and reduce neuronal excitability. Their depletion increases risk for seizures. Hypomagnesemia can be due to inadequate dietary intake of magnesium-containing food, chronic diarrhea, and renal tubular dysfunction. Hypomagnesemia contributes to the dysregulation of the parathyroid hormone release and generates persistence of hypocalcemia, which, in turn, contributes to decreased reabsorption of phosphate in the proximal tubule, worsening the hypophosphatemia. Finally, vitamin D deficiency, one of the most common vitamin disorders worldwide, leads to hypocalcemia and is often found in chronic alcoholics. ${ }^{40}$

This population is at higher risk of developing ODS due to the complex interplay of electrolytes and metabolic abnormalities leading to multiple organ dysfunction. The recommendations for treatment include volume assessment and diagnosis of all electrolyte abnormalities. Infusion of dextrose $5 \%$ in $0.9 \%$ normal saline would be indicated if ketoacidosis, hypovolemia, and hypoglycemia are initially diagnosed. Restoration of intrinsic insulin release and correction of volume are essential, but insulin infusion is rarely necessary. Thiamine supplementation is highly recommended as it is often deficient in these patients, and its infusion can prevent Wernicke's encephalopathy or Korsakoff's syndrome. Awareness of possible ingestion of other alcohols, such as methanol, ethylene glycol, and isopropanol, is important. Finally, if hyponatremia has not corrected itself with these measures, hypertonic saline infusion can be considered. Severe hyponatremia associated with neurological symptoms can be corrected initially with small boluses of hypertonic saline and is safe.

\section{Conclusions}

1. A good history and assessment of all risk factors, chronic medical conditions, and medication are essential when managing a patient with hyponatremia and neurological symptoms.

2. Osmotic demyelination syndrome is a rare complication of rapid correction of hyponatremia; it can be severe and deadly but is often reversible.

3. Osmotic demyelination syndrome should be suspected in post-operative patients with nausea, vomiting, and headache not resolving with antiemetic and analgesic drugs, particularly if they develop new neurological symptoms, including dysphagia or other bulbar symptoms.

4. Patients with chronic alcohol use are at higher risk of developing ODS, due to poor oral intake leading to multiple electrolytes abnormalities and impaired metabolism. These patients should receive thiamine, and all electrolytes and osmoles should be assessed promptly.

5. Guidelines for treatment are starting to emerge. Hypertonic saline solution infusion in small boluses of $150 \mathrm{ml}$, with the goal of increasing sodium levels to $5-6 \mathrm{mEq} / \mathrm{L}$ in the first $5 \mathrm{hrs}$, and up to $15-20 \mathrm{mEq} / \mathrm{L}$ in $48 \mathrm{hrs}$ is safe in most cases of true severe hyponatremia. 
Article citation: Gagnon DG. Hyponatremia, osmotic demyelination syndrome, and the importance of the patient's history. The Southwest Respiratory and Critical Care Chronicles 2021;9(37):45-53

From: Department of Family Medicine, Texas Tech University Health Sciences Center, Lubbock, Texas

Submitted: $11 / 1 / 2020$

Accepted: 12/29/2020

Reviewer: Camilo Pena MD

Conflicts of interest: none

This work is licensed under a Creative Commons Attribution-ShareAlike 4.0 International License.

\section{REFERENCES}

1. Bagshaw SM, Townsend DR, McDermid RC. Disorders of sodium and water balance in hospitalized patients. Can J Anaesth 2009;56:151-67.

2. Funk GC, Lindner G, Druml W, et al. Incidence and prognosis of dysnatremias present on ICU admission. Intensive Care Med 2010;36:304-11.

3. Mohan S, Gu S, Parikh A, Radhakrishnan J. Prevalence of hyponatremia and association with mortality: results from NHANES. Am J Med 2013;126:1127-37 e1.

4. Schmidt BM. [The most frequent electrolyte disorders in the emergency department : what must be done immediately?]. Internist (Berl) 2015;56:753-9.

5. Ghali JK. Mechanisms, risks, and new treatment options for hyponatremia. Cardiology 2008;111:147-57.

6. Singh TD, Fugate JE, Rabinstein AA. Central pontine and extrapontine myelinolysis: a systematic review. Eur J Neurol 2014;21:1443-50.

7. Woodfine JD, Sood MM, MacMillan TE, et al. Derivation and validation of a novel risk score to predict overcorrection of severe hyponatremia: The Severe Hyponatremia Overcorrection Risk (SHOR) Score. Clin J Am Soc Nephrol 2019; 14: 975-82.

8. Adams RD, Victor M, Mancall EL. Central pontine myelinolysis: a hitherto undescribed disease occurring in alcoholic and malnourished patients. AMA Arch Neurol Psychiatry 1959;81:154-72.

9. George JC, Zafar W, Bucaloiu ID, et al. Risk factors and outcomes of rapid correction of severe hyponatremia. Clin J Am Soc Nephrol 2018;13:984-92.

10. Lambeck J, Hieber M, Dressing A, et al. Central pontine myelinosis and osmotic demyelination syndrome. Dtsch Arztebl Int 2019;116:600-6.
11. Hew-Butler T, Rosner MH, Fowkes-Godek S, et al. Statement of the Third International Exercise-Associated Hyponatremia Consensus Development Conference, Carlsbad, California, 2015. Clin J Sport Med 2015;25:303-20.

12. Aegisdottir H, Cooray $C$, Wirdefeldt $K$, et al. Incidence of osmotic demyelination syndrome in Sweden: A nationwide study. Acta Neurol Scand 2019;140:342-9.

13. Lohr JW. Osmotic demyelination syndrome following correction of hyponatremia: association with hypokalemia. Am J Med 1994;96:408-13.

14. Ahmed A, Tschetter PA, Krasowski MD, et al. Massive ethylene glycol poisoning triggers osmotic demyelination syndrome. J Emerg Med 2014;46:e69-74.

15. Chinoy A, Wright NB, Bone M, et al. Severe hypokalaemia in diabetic ketoacidosis: a contributor to central pontine myelinolysis? Endocrinol Diabetes Metab Case Rep 2019; 2019.

16. Hsieh $\mathrm{HC}, \mathrm{Wu} \mathrm{SH}$, Chiu $\mathrm{CC}$, et al. Excessive sodium bicarbonate infusion may result in osmotic demyelination syndrome during treatment of diabetic ketoacidosis: a case report. Diabetes Ther 2019;10:765-71.

17. Jha AA, Behera V, Jairam A, et al. Osmotic demyelination syndrome in a normonatremic patient of chronic kidney disease. Indian J Crit Care Med 2014;18:609-11.

18. Pham PM, Pham PA, Pham SV, et al. Correction of hyponatremia and osmotic demyelinating syndrome: have we neglected to think intracellularly? Clin Exp Nephrol 2015; 19:489-95.

19. Baek TH, Yang SH, Sung JH, et al. Central pontine and extrapontine myelinolysis in a patient with traumatic brain injury following not rapid correction of hyponatremia: a case report. Korean J Neurotrauma 2014;10:31-4.

20. Bansal LR, Zinkus T. Osmotic demyelination syndrome in children. Pediatr Neurol 2019;97:12-7.

21. de Souza A, Desai PK. More often striatal myelinolysis than pontine? A consecutive series of patients with osmotic demyelination syndrome. Neurol Res 2012;34:262-71.

22. Giuliani C, Peri A. Effects of hyponatremia on the brain. J Clin Med 2014;3:1163-77.

23. Kim DK, Joo KW. Hyponatremia in patients with neurologic disorders. Electrolyte Blood Press 2009;7:51-7.

24. Gamba G. Molecular physiology and pathophysiology of electroneutral cation-chloride cotransporters. Physiol Rev 2005;85:423-93.

25. Jentsch TJ. VRACs and other ion channels and transporters in the regulation of cell volume and beyond. Nat Rev Mol Cell Biol 2016;17:293-307.

26. Kahle KT, Khanna AR, Alper SL, et al. K-Cl cotransporters, cell volume homeostasis, and neurological disease. Trends Mol Med 2015;21:513-23. 
27. Mongin AA. Volume-regulated anion channel-a frenemy within the brain. Pflugers Arch 2016;468:421-41.

28. Mongin AA, Orlov SN. Mechanisms of cell volume regulation and possible nature of the cell volume sensor. Pathophysiology 2001;8:77-88.

29. Pedersen SF, O'Donnell ME, Anderson SE, et al. Physiology and pathophysiology of $\mathrm{Na}+/ \mathrm{H}+$ exchange and $\mathrm{Na}+-\mathrm{K}+$ -2Cl- cotransport in the heart, brain, and blood. Am J Physiol Regul Integr Comp Physiol 2006;291:R1-25.

30. Huda MS, Boyd A, Skagen K, et al. Investigation and management of severe hyponatraemia in a hospital setting. Postgrad Med J 2006;82:216-9.

31. Whyte M, Down C, Miell J,et al. Lack of laboratory assessment of severe hyponatraemia is associated with detrimental clinical outcomes in hospitalised patients. Int J Clin Pract 2009;63:1451-5.

32. Rondon-Berrios H, Berl T. Mild chronic hyponatremia in the ambulatory setting: significance and management. Clin J Am Soc Nephrol 2015;10:2268-78.

33. Diringer MN, Zazulia AR. Hyponatremia in neurologic patients: consequences and approaches to treatment. Neurologist 2006;12:117-26.

34. Ellison DH, Berl T. Clinical practice. The syndrome of inappropriate antidiuresis. N Engl J Med 2007;356:2064-72.

35. Ramirez E, Rodriguez A, Queiruga J, et al. Severe hyponatremia is often drug induced: 10-year results of a prospective pharmacovigilance program. Clin Pharmacol Ther 2019;106:1362-79.

36. Berl T. Vasopressin antagonists. N Engl J Med 2015;372: 2207-16.

37. Soupart A, Coffernils M, Couturier B, et al. Efficacy and tolerance of urea compared with vaptans for long-term treatment of patients with SIADH. Clin J Am Soc Nephrol 2012; 7:742-7.

38. Gankam Kengne F, Soupart A, Pochet R, et al. Re-induction of hyponatremia after rapid overcorrection of hyponatremia reduces mortality in rats. Kidney Int 2009;76:614-21.

39. Ayus JC, Moritz ML. Misconceptions and barriers to the use of hypertonic saline to treat hyponatremic encephalopathy. Front Med (Lausanne) 2019;6:47.

40. Holick MF. The vitamin D deficiency pandemic: Approaches for diagnosis, treatment and prevention. Rev Endocr Metab Disord 2017;18:153-65.

41. Palmer BF, Clegg DJ. Electrolyte disturbances in patients with chronic alcohol-use disorder. N Engl J Med 2017;377: 1368-77.

42. De Marchi S, Cecchin E, Basile A, et al. Renal tubular dysfunction in chronic alcohol abuse-effects of abstinence. N Engl J Med 1993;329:1927-34.

43. Huang CL, Kuo E. Mechanism of hypokalemia in magnesium deficiency. J Am Soc Nephrol 2007;18:2649-52. 